\title{
Surface dynamics of a thin polystyrene film probed by low-energy muons
}

\author{
F. L. Pratt \\ ISIS Facility, Rutherford Appleton Laboratory, Chilton, Oxfordshire OX11 0QX, United Kingdom \\ T. Lancaster, M. L. Brooks, and S. J. Blundell \\ University of Oxford, Department of Physics, Clarendon Laboratory, Parks Road, Oxford OXI 3PU, United Kingdom \\ T. Prokscha, E. Morenzoni, A. Suter, H. Luetkens, R. Khasanov, R. Scheuermann, and U. Zimmermann \\ Paul Scherrer Institut, CH-5232 Villigen PSI, Switzerland \\ K. Shinotsuka and H. E. Assender \\ University of Oxford, Department of Materials, Parks Road, Oxford OX1 3PH, United Kingdom \\ (Received 12 May 2005; revised manuscript received 15 July 2005; published 13 September 2005)
}

\begin{abstract}
Low-energy muons have been used to probe the local motion in a thin-film sample of polystyrene as a function of depth below the free surface. The muon spin relaxation in zero magnetic field is dominated by a muoniated radical state which is formed on the polystyrene ring. The muon spin-relaxation rate $\lambda$ is found to be highly sensitive to the dynamical state of the polymer, and $\lambda$ provides a local probe of changes in the dynamical correlation time around the glass transition. When high-energy muons are used to study the bulk properties, the glass-transition temperature $T_{g}$ is revealed as a change in the temperature dependence of $\lambda$. Reducing the muon implantation depth, while holding the sample a few degrees below the bulk $T_{g}$, leads to a drop in $\lambda$, signifying a reduction in the local $T_{g}$ on approaching the surface. The width of the dynamical surface layer is found to be enhanced over previous estimates made at much lower temperatures, providing experimental evidence for critical divergence of the layer width on approaching the bulk $T_{g}$.
\end{abstract}

DOI: $10.1103 /$ PhysRevB.72.121401

PACS number(s): 68.47.Mn, 68.35.Ja, 68.35.Rh, 76.75.+i

It has recently become appreciated that the properties of a polymer near a surface or interface can be significantly different from the properties of the bulk. ${ }^{1}$ The dynamical properties, most readily characterized by the glass-transition temperature $T_{g}$, show dramatic changes when the volume fraction of the surface region is increased by reducing the overall thickness of a film. Optical techniques such as ellipsometry and Brillouin scattering have been important for establishing the dependence of $T_{g}$ on film thickness $h$ (Refs. 2,3 ), with polystyrene (PS) providing the focus for the studies. For longer chain PS, confinement dominates when $h$ becomes shorter than the end-to-end distance of a polymer chain $R_{E E}$ (Ref. 3). Shorter chains are less affected by confinement and may show an intrinsic mobility enhancement associated with the free surface of the film. For low molecular weight, free-standing PS films, the $T_{g}$ suppression from the bulk value $T_{g}^{\text {bulk }}$ can become particularly significant for $h$ below $50 \mathrm{~nm}$; e.g., for $h=21.6 \mathrm{~nm}, \Delta T_{g}=T_{g}^{\text {bulk }}-T_{g}$ was reported at $58 \mathrm{~K}$ (Ref. 4). Although various different studies have followed these initial reports, full consensus on the effect has not yet been reached. This may at least partly reflect differences between probes in their spatial averaging and their sensitivity to different dynamical time scales, as well as reflecting temperature dependence of the size of the dynamical surface region itself.

Local dynamical probes are useful to gain further insight into these effects; e.g., some studies of PS films using techniques such as atomic force microscopy ${ }^{5}$ and fluorescence labeling ${ }^{6}$ have seen similar $\Delta T_{g}$ to those reported in earlier optical studies. Probes that allow the depth profile of the dynamical properties to be studied directly are particularly valuable. Positron annihilation provides such a technique and a recent depth-resolved study showed a clear depression of $T_{g}$ as the surface was approached $;^{7} T_{g}$ was reduced by $57 \mathrm{~K}$ at a mean implantation depth of $5 \mathrm{~nm}$ and by $19 \mathrm{~K}$ at a depth of $24 \mathrm{~nm}$. Another local probe technique with depth resolution on the nm scale has emerged very recently, based on the implantation of low-energy muons (LEM) (Ref. 8). We report here the use of this technique to study dynamical properties near the surface of a thin PS film.

The PS used in these measurements was obtained from Polymer Source Inc. $\left(M_{w}=62600, M_{w} / M_{n}=1.04\right)$ and a thin film was prepared on a copper substrate by spin coating at $2000 \mathrm{rpm}$ using a $15 \%$ solution of the polymer in cyclohexanone. A thickness of $0.46 \mu \mathrm{m}$ was estimated from ellipsometry of films prepared under identical conditions on glass substrates. Since $R_{E E}$ for this molecular weight of PS is 16 $\mathrm{nm}$, no significant confinement effect is expected at this thickness and the majority of the film away from the surface layer region should behave as bulk.

This work was performed at the $\mathrm{S} \mu \mathrm{S}$, Paul Scherrer Institut, Switzerland. The main measurements used the lowenergy muon (LEM) instrument, ${ }^{9}$ while additional bulk measurements were made at higher muon energies $(3.7 \mathrm{MeV}$ ) using the DOLLY and GPD instruments. For the LEM measurements the initial spin polarization of the implanted muon was parallel to the plane of the film. Experiments were either in zero magnetic field $(\mathrm{ZF})$ or with a transverse calibration field (TF) perpendicular to the plane of the film. The time evolution of the polarization of the muon ensemble was measured with four positron emission detectors for $\mathrm{TF}$ or two detectors for ZF. The implantation energy of the muons was 


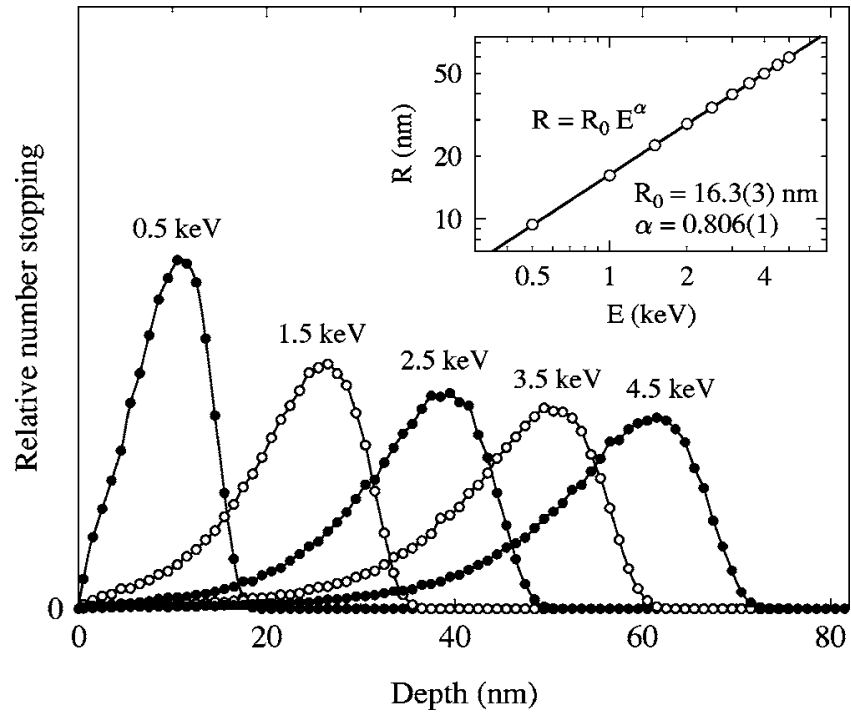

FIG. 1. The stopping profile calculated for slow muons with different implantation energies in PS using TRIM.SP. The inset shows the mean range $R$ vs implantation energy $E$. $R$ can be expressed as a power law in $E$, with the fit parameters as shown, when $E$ is expressed in $\mathrm{keV}$.

varied between 5.15 and $2.2 \mathrm{keV}$ by varying the target potential. Using the Monte Carlo program TRIM.SP (Ref. 10), which has been shown to accurately describe the stopping profile of slow muons, ${ }^{11}$ the range of the slow muons in PS was estimated for different implantation energies (Fig. 1). This shows that the depth range probed here covers the region from $70 \mathrm{~nm}$ down to below $30 \mathrm{~nm}$, spanning a range of thickness over which significant $\Delta T_{g}$ was observed in earlier studies.

Previous muon studies on bulk PS using fields of a few $\mathrm{kG}$ (Ref. 12) have shown that muonium forms rapidly, reacting with the phenyl ring in the polymer to form a muoniated radical state; this radical is a sensitive probe of the dynamical state of the polymer. Such high fields are not available at the PSI LEM facility; the current apparatus can make measurements in ZF and relatively small TF, so the majority of the measurements have been made in the ZF configuration. At temperatures above $T_{g}$ the muon spin relaxation is dominated by the dynamical properties of the radical. In this case the measured relaxation rate $\lambda$ is proportional to the reorientational correlation time of the ring, which is in turn coupled to the segmental dynamics of the polymer chain.

Examples of ZF muon spin relaxation in PS are shown in Fig. 2. In all the measurements a very fast relaxation takes place within a small fraction of a microsecond. This is associated with the radical state formed in PS by muon implantation. Both standard $3.7 \mathrm{MeV}$ muons and $4.15 \mathrm{keV}$ slow muons probe deeply enough to measure the bulk, and a consistent value for the relaxation rate of the radical signal in the bulk is obtained using both energies. However, it can be seen in Fig. 2 that the fraction of the fast relaxing radical signal is significantly enhanced in the slow muon case. Although the exact mechanism for this is not yet clear, we note that differences in the paramagnetic state fraction between lowenergy and high-energy muons have also been seen in sev-

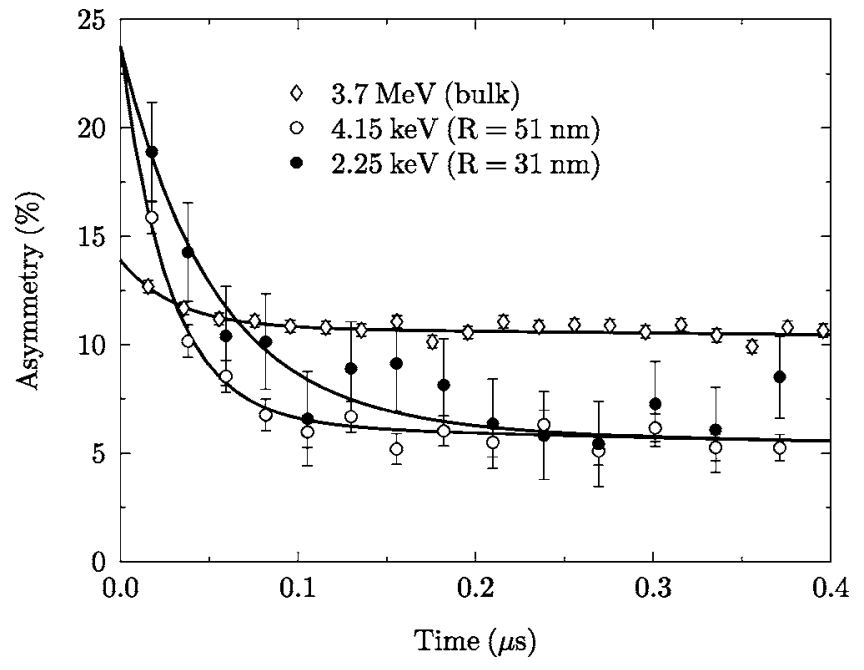

FIG. 2. The ZF muon spin relaxation for standard high-energy (3.7 MeV) muons at $300 \mathrm{~K}$ and for low-energy muons with two different implantation energies: deeply implanted at $4.15 \mathrm{keV}$ and more shallowly implanted at $2.25 \mathrm{keV}$. For deep implantation, the relaxation does not vary significantly with temperature below $T_{g}^{\text {bulk }}=378 \mathrm{~K}$ and the data are the average of several runs within the glass phase. The shallow implantation run is measured at $T_{q}$ $=373 \mathrm{~K}=T_{g}^{\text {bulk }}-5 \mathrm{~K}$ and shows a reduced relaxation rate that is characteristic of the bulk relaxation at a somewhat higher temperature, suggesting the presence of a high mobility surface region.

eral other insulating systems. ${ }^{13}$ The enhanced radical signal observed here is certainly beneficial for these dynamical studies using slow muons. From earlier studies of muon spin rotation and relaxation in PS (Refs. 12,14), the isotropic hyperfine parameter $A$ was found to be in the region of 500 $\mathrm{MHz}$ and the dipolar contribution to the hyperfine tensor was estimated to be in the region of $10-15 \mathrm{MHz}$ (Refs. 12,14).

A comparison is made in Fig. 2 between the relaxation in the glassy state of the bulk measured with both $3.7 \mathrm{MeV}$ and $4.15 \mathrm{keV}$ muons and the relaxation observed close to the surface of the polymer with $2.25 \mathrm{keV}$ muons at $T_{q}$, a temperature $5 \mathrm{~K}$ below $T_{g}^{\text {bulk }}$. The significantly slower relaxation seen near the surface can be interpreted in terms of a reduced $T_{g}$ in the surface region. The data are well fitted by a single exponential relaxation function; the initial asymmetry and weakly relaxing asymmetry baseline are determined by global fits to the combined data set for all zero-field runs. This leaves the single parameter of the relaxation rate to be fitted in the scans that have been made of the temperature dependence and the implantation depth dependence of the relaxation. Such a constrained fitting approach is essential to make optimum use of the slow muon data, whose counting statistics are inevitably much weaker than those of standard muon relaxation experiments, due to the low efficiency of the muon slowing down process. ${ }^{9}$

The temperature dependence of the relaxation using higher-energy muons to probe the bulk polymer is shown in Fig. 3. The glass transition is seen as a crossover from essentially temperature-independent behavior in the glassy state to a strong temperature dependence above $T_{g}$ where the relaxation rate falls off with increasing temperature. This reflects a motional narrowing regime above $T_{g}$ where the re- 


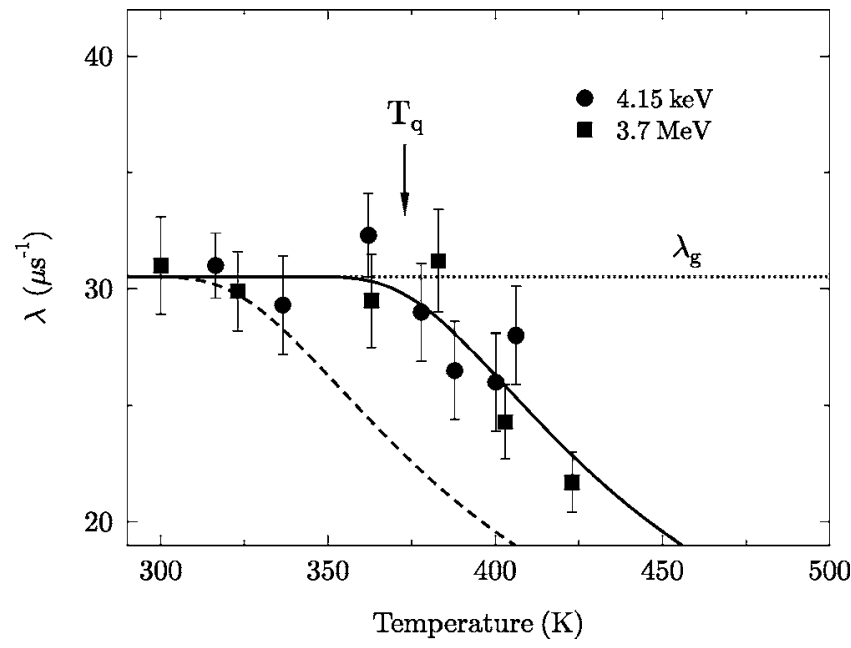

FIG. 3. Temperature-dependent ZF relaxation rate measured for the bulk polymer using both standard energy $3.7 \mathrm{MeV}$ muons and deeply implanting $4.15 \mathrm{keV}$ slow muons. The relaxation rate starts to fall on warming the sample above the bulk glass-transition temperature. The data are consistent with (1)-(3) (solid line). The dashed line shows the expected curve for $T_{g}$ suppressed by $50 \mathrm{~K} . T_{q}$ is chosen to give good sensitivity to local $T_{g}$ changes in a depthdependent scan.

laxation rate becomes proportional to a motional correlation time $\tau$ which gets shorter as the temperature increases. The relaxation in this situation can be described by the expression

$$
\lambda=2 \Delta^{2} \tau
$$

Here $\Delta$ is assumed to be independent of temperature and reflects the coupling mechanism between the local motion of the polymer and the muon spin. For relaxation mechanisms involving modulation of the orientation of the hyperfine tensor of the radical, $\Delta$ is expected to be closely related to the anisotropic dipolar coupling, ${ }^{15}$ i.e., it should be of order 10 $\mathrm{MHz}$ (Ref. 12). The correlation time $\tau$ is assumed to be described by the form

$$
\tau^{-1}=\tau_{g}^{-1}+\left(C / \tau_{0}\right)\left(\tau_{s} / \tau_{0}\right)^{-\alpha}
$$

The first term in (2) describes the relaxation seen in the glass phase $\lambda_{g}\left(=2 \Delta^{2} \tau_{g}\right)$ and the second term describes the coupling of the muon to the segmental dynamics, characterized by $\tau_{s}$, that shows strong temperature dependence. This coupling is defined by the dimensionless parameters $C$ and $\alpha ; \tau_{0}$ is a reference time, taken to be the value of $\tau_{s}$ at $T_{g}$, i.e., 100 s. If $C$ and $\alpha$ are both 1 , the coupling is direct, whereas reduced values of $C$ and $\alpha$ are expected for the muon probe, reflecting the indirect coupling mechanism between phenyl ring rotation and the segmental motion of the polymer backbone. This type of power-law scaling has previously been seen for indirect probes of glass dynamics using chromophores and spin labels. ${ }^{17}$ While many different ways of modeling $\tau_{s}$ are possible, we choose here the simple approach of assuming the Williams-Landel-Ferry (WLF) form, ${ }^{16}$

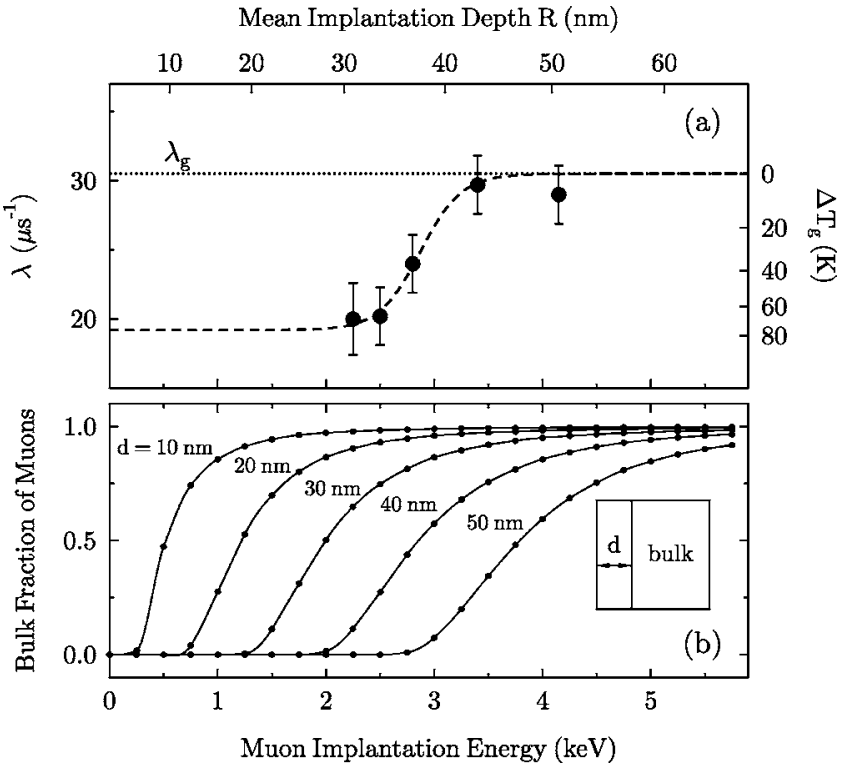

FIG. 4. (a) Dependence of the ZF muon spin-relaxation rate on the implantation energy and corresponding mean implantation depth $R$ (filled circles). The sample temperature was held at $T_{q}=373 \mathrm{~K}$. The data suggest that a surface region with a depth of around $35 \mathrm{~nm}$ remains in a highly dynamic state while the bulk polymer would be in the glass state at the same temperature. (b) The fraction of muons stopping in the bulk according to the TRIM.SP simulation is shown for several different surface layer thicknesses $d$. The observed transition width is seen to be consistent with calculation.

$$
\log \left(\tau_{s} / \tau_{0}\right)=\frac{-C_{1}\left(T-T_{g}\right)}{C_{2}+T-T_{g}}
$$

The WLF parameters take the characteristic values $C_{1}=17$ and $C_{2}=50 \mathrm{~K}$. Fitting the temperature-dependent data to (1)-(3) with $\Delta=10 \mathrm{MHz}$ gives $\lambda_{g}=30.5(8) \mu \mathrm{s}^{-1}, \quad \alpha$ $=0.11(8)$, and $C=0.5(3)$. It can be seen in Fig. 3 that this simple model provides a good parametrization of the temperature dependence. Reducing $T_{g}$ in this model shifts the knee in the relaxation rate curve to lower temperature (Fig. 3, dashed line).

Since the relaxation rate is reduced compared to the bulk value when $T>T_{g}$, fixing the temperature to $T_{q}=373 \mathrm{~K}$ provides good sensitivity to detect any reduction in the local $T_{g}$ as the implantation depth is varied. Such a reduced $T_{g}$ would lead to a detectable reduction in $\lambda$ (e.g., following the dashed curve in Fig. 3). The result of a scan of implantation depth under these conditions is shown in Fig. 4(a), where the relaxation rate is indeed seen to decrease quite sharply for implantation energies below $3 \mathrm{keV}$. The midpoint of the transition is around $35 \mathrm{~nm}$ with a width that is consistent with the calculated muon stopping profile [Fig. 4(b)]. The data suggest that the bulk behavior at $T_{q}$ extends to within $35 \mathrm{~nm}$ of the surface and that a surface layer exists below this depth with dynamical behavior that differs significantly from the bulk. The parametrization of $\lambda$ using (1)-(3) suggests that the reduced $\lambda$ seen at $30 \mathrm{~nm}$ would be expected when $T_{g}$ is reduced by $70 \mathrm{~K}$, implying that this is the $\Delta T_{g}$ of the surface region [Fig. 4(a), right-hand scale]. 
The size of $\Delta T_{g}$ for the surface region is consistent with that inferred from Brillouin scattering studies of freestanding films. ${ }^{4}$ Our observed length scale is, however, notably larger than the values inferred from modeling $\Delta T_{g}$ data, which typically lie within the range $2-10 \mathrm{~nm}$ (Ref. 1). This apparent difference can be understood by consideration of the temperature dependence of $d$, the width of a dynamical surface layer, which is expected to diverge as the bulk glasstransition temperature is approached (Fig. 5), ${ }^{2,18}$ following the relation

$$
d=h_{0} /\left(T_{g}^{\mathrm{bulk}} / T-1\right)^{\nu} .
$$

A recent temperature-dependent NMR study on a suspension of 100-nm-diam droplets gave the length parameter $h_{0}$ $=0.47 \mathrm{~nm}$ with $\nu=1$ (Ref. 18). Note that in our measurement $T_{q}$ is very close to $T_{g}^{\text {bulk }}$ and significant enhancement of $d$ is expected. From our data using (4) with $\nu=1$, we estimate $h_{0}=0.5(2) \mathrm{nm}$. Further estimates of $h_{0}$ can be obtained from fits of the $h$ dependence of $T_{g}$ in thin films using $T_{g}$ $=T_{g}^{\text {bulk }} /\left(1+\left(h_{0} / h\right)^{1 / \nu}\right)$, which follows from setting $d=h$ in $(4)$. Estimates are 0.68 (Ref. 19), 0.83 (Ref. 20), and $0.82 \mathrm{~nm}$ (Ref. 21), only slightly larger than our value and that of the NMR study. ${ }^{18}$

These studies of a polymer surface using implanted slow muons have clearly demonstrated the potential of the technique as a depth-controllable dynamical probe. The results are in accord with a picture in which $T_{g}$ depression is a

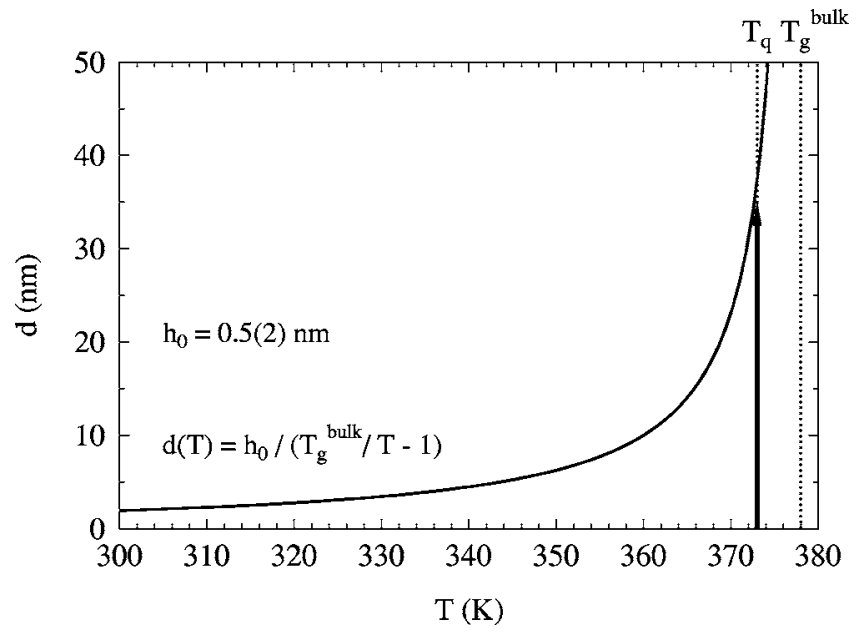

FIG. 5. Expected divergence of the effective width of the high mobility surface region as the bulk glass transition is approached. The temperature dependence of the width is determined by $T_{g}^{\text {bulk }}$ and the length parameter $h_{0}$. From the 35 -nm width observed at the probe temperature $T_{q}, h_{0}=0.5(2) \mathrm{nm}$ is estimated.

property of a free surface of the polymer, where the local segmental mobility is significantly enhanced. The observed depth of the enhanced mobility surface layer is consistent with models of surface dynamics which allow for divergence of the layer width on approaching $T_{g}^{\text {bulk }}$ (Ref. 18).
${ }^{1}$ J. A. Forrest and K. Dalnoki-Veress, Adv. Colloid Interface Sci. 94, 167 (2001).

${ }^{2}$ J. L. Keddie, R. A. L. Jones, and R. A. Cory, Europhys. Lett. 27, 59 (1994).

${ }^{3}$ J. A. Forrest, K. Dalnoki-Veress, J. R. Stevens, and J. R. Dutcher, Phys. Rev. Lett. 77, 2002 (1996); 77, 4108 (1996).

${ }^{4}$ J. Mattsson, J. A. Forrest, and L. Börjesson, Phys. Rev. E 62, 5187 (2000).

${ }^{5}$ V. N. Bliznyuk, H. E. Assender, and G. A. D. Briggs, Macromolecules 35, 6613 (2002); K. Akabori, K. Tanaka, T. Kajiyama, and A. Takahara, ibid. 36, 4937 (2003).

${ }^{6}$ C. J. Ellison and J. M. Torkelson, Nat. Mater. 2, 695 (2003).

${ }^{7}$ Y. C. Jean, R. Zhang, H. Cao, J.-P. Yuan, C.-M. Huang, B. Nielson, and P. Asoka-Kumar, Phys. Rev. B 56, R8459 (1997).

${ }^{8}$ P. Bakule and E. Morenzoni, Contemp. Phys. 45, 203 (2004).

${ }^{9}$ E. Morenzoni, in Muon Science, edited by S. L. Lee, S. H. Kilcoyne, and R. Cywinski (IOP, Bristol and Philadelphia, 1999).

${ }^{10} \mathrm{~W}$. Eckstein, Computer Simulation of Ion-Solid Interactions (Springer-Verlag, Berlin, 1991).

${ }^{11}$ E. Morenzoni, H. Glückler, T. Prokscha, R. Khasanov, H. Luetkens, M. Birke, E. M. Forgan, C. Niedermayer, and M. Pleines, Nucl. Instrum. Methods Phys. Res. B 192, 254 (2002).
${ }^{12}$ F. L. Pratt, S. J. Blundell, I. M. Marshall, T. Lancaster, A. Husmann, C. Steer, W. Hayes, C. Fischmeister, R. E. Martin, and A. B. Holmes, Physica B 326, 34 (2003).

${ }^{13}$ T. Prokscha, E. Morenzoni, N. Garifianov, H. Glückler, R. Khasanov, H. Luetkens, and A. Suter, Physica B 326, 51 (2003).

${ }^{14}$ The dipolar coupling reported in the preceding reference has been normalized by a factor of $\sqrt{2}$ to be consistent with the definition (1) used in this paper.

${ }^{15}$ B. W. Lovett, S. J. Blundell, J. S. Stiessberger, F. L. Pratt, Th. Jestädt, W. Hayes, S. P. Cottrell, and I. D. Reid, Phys. Rev. B 63, 054204 (2001).

${ }^{16}$ M. L. Williams, R. F. Landel, and J. D. Ferry, J. Am. Chem. Soc. 77, 3701 (1955).

${ }^{17}$ L. Andreozzi, M. Faetti, M. Giordano, and D. Leporini, J. Phys.: Condens. Matter 11, A131 (1999).

${ }^{18}$ S. Herminghaus, R. Seemann, and K. Landfester, Phys. Rev. Lett. 93, 017801 (2004).

${ }^{19}$ J. H. Kim, J. Jang, and W-C. Zin, Langmuir 16, 4064 (2000).

${ }^{20}$ S. Kawana and R. A. L. Jones, Phys. Rev. E 63, 021501 (2001).

${ }^{21}$ S. Herminghaus, K. Jacobs, and R. Seemann, Eur. Phys. J. E 5, 531 (2001). 